\title{
OPTIMIZATION OF COMPOSITE STRUCTURES SUBJECT TO LOCAL STRESS CONSTRAINTS
}

By

\section{Robert Lipton}

and

Michael Stuebner

IMA Preprint Series \# 2043

(May 2005)

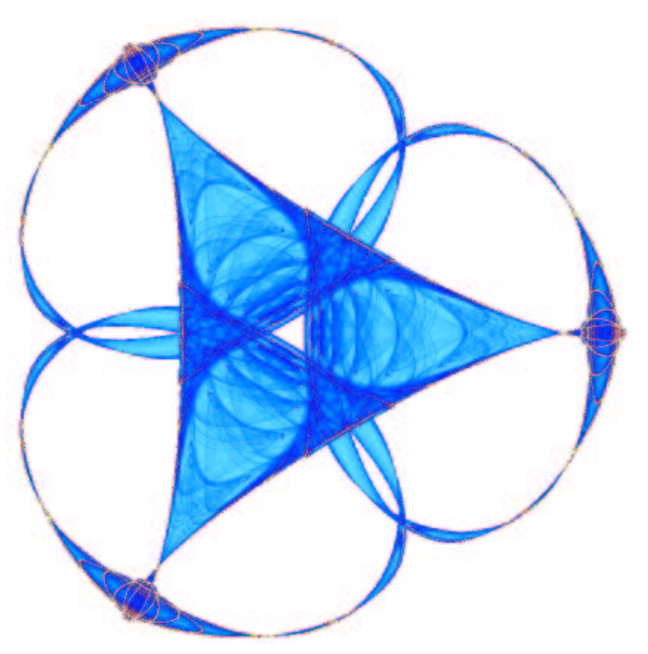

INSTITUTE FOR MATHEMATICS AND ITS APPLICATIONS

UNIVERSITY OF MINNESOTA

514 Vincent Hall

206 Church Street S.E.

Minneapolis, Minnesota 55455-0436

Phone: 612/624-6066 Fax: 612/626-7370

URL: http://www.ima.umn.edu 


\title{
Optimization of composite structures subject to local stress constraints
}

\author{
Robert Lipton ${ }^{1}$ and Michael Stuebner \\ Mathematics Department, Louisiana State University, \\ Baton Rouge, Louisiana 70803
}

\begin{abstract}
An extension of current methodologies is introduced for optimization of graded microstructure subject to local stress criteria. The method is based on new multiscale stress criteria given by macrostress modulation functions. The modulation functions quantify the intensity of local stress fluctuations at the scale of the microstructure due to the imposed macroscopic stress. The methodology is illustrated for long cylindrical shafts reinforced with stiff cylindrical elastic fibers with generators parallel to the shaft. Examples are presented for shaft cross sections that possess reentrant corners typically seen in lap joints and junctions of struts. It is shown that the computational methodology delivers graded fiber microgeometries that provide overall structural rigidity while at the same time tempering the influence of stress concentrations near reentrant corners.
\end{abstract}

Keywords: Stress constraints, optimal structural design

\section{Introduction}

The high specific stiffness of composite materials makes them attractive structural materials for use in aerospace applications. These materials are increasingly being used in geometries that involve abrupt dimensional changes within structural components, such as skins connected to ribs, panel reinforcements and junctions of struts. These geometries contain stress concentrations and thus present potential sources for structural failure. In this paper we present a computational design method for grading the microstructure in order to control local stress in the vicinity of stress concentrations. The method is based upon new rigorous multiscale stress criteria that connect the macroscopic or homogenized stress to local stress fluctuations at the scale of the microstructure [25, 26, 27].

It is now well known that effective constitutive properties relating average stress to average strain can be employed in the numerical design of composite structures for optimal structural compliance and natural frequency. This type of design problem has received significant attention from both the applied mathematics and structural optimization communities in the 1980s and 1990s $[1,4,7,10,11,12,14,17,18,31,32,35]$. This list of references is by no means complete and the reader is referred to the reviews and manuscripts $[2,5,8,9,19,30,33,37]$. The associated body of methods are referred to as the homogenization approach to structural optimization. These treatments have focused on the design problem in the absence of stress or strain constraints.

\footnotetext{
${ }^{1}$ Corresponding Author: E-mail address: lipton@math.lsu.edu, Phone:+1-6176-538782, Fax:+1-2255784276
} 
Recent efforts have initiated the development of numerical methods for structural optimization in the presence of stress constraints. The investigation given in [13] provides a numerical method for the stress constrained minimum volume design problem. The method is carried out using an empirical model that is an extension of the power penalized stiffness model also known as the Solid Isotropic Microstructure with Penalization (SIMP) model [5]. The choice of local stress constraints proposed in [13] is motivated by the explicit form of the corrector tensors associated with rank two orthogonal laminar microstructures. Other work has extended the numerical methodology providing the opportunity for design of composites and topology in the presence of mean square stress constraints. The problem of mean square stress constrained structural optimization for fiber reinforced shafts is taken up in [20]. In that work a numerical algorithm is developed based on a suitable homogenized quantity (the covariance tensor) that accurately encodes the mean square stress constraints. The work of [3] introduces a partial relaxation for topology optimization for minimum mean square stress using finite rank laminates. The rigorous theoretical context behind the homogenization approach to mean square stress (or gradient) constrained structural optimization has been worked out in [23], [24]. Other recent theoretical and numerical developments have addressed problems of minimizing objective functions depending explicitly on the mean square norm of the gradients of the state variable [15, 22, 34, 36, 38].

In this treatment we consider design problems that require point wise constraints on the local stress. Suitable multiscale quantities beyond effective constitutive laws are used to accurately enforce local stress criteria. These quantities dubbed macrostress modulation functions are introduced in $[25,26,27]$. The design formulation given here is expressed in terms of a homogenized design problem that satisfies two requirements: The first is that the homogenized design problem is computationally tractable. The second is that the solution of the homogenized design problem provides the means to identify graded microstructures that deliver the required structural response while at the same time provide local stress control. The approach put forth in this paper is in essence an inverse method that uses new tools from homogenization theory to identify optimal graded microgeometries. Because of this we will refer to it as an inverse homogenization design method. The rigorous theoretical basis for this approach is given in [21] and [29].

To illustrate the ideas this article treats the problem of reinforcement of a long shaft with constant cross section subjected to torsion loading. The microstructure within the shaft consists of long reinforcement fibers of constant cross section with isotropic shear modulus $G_{f}$ embedded in a more compliant material with shear modulus $G_{m}$. The shaft together with the fibers are right cylinders with generators along the $x_{3}$ axis. The cross section of the reinforced shaft is specified by the region $\Omega$ in the $x_{1}-x_{2}$ plane. In the neighborhood of any point $\mathbf{x}=\left(x_{1}, x_{2}\right)$ the local microgeometry is given by a periodic geometry with period cell filled with the Hashin Shtrikman coated cylinder assemblage [16], see Figure 1. The characteristic length scale of the period relative to the size of the design domain is taken to be small and is denoted by $\varepsilon$. The coated cylinder assemblage corresponds to stiff fibers of circular cross section separated by compliant matrix material. The local area fraction of the fiber phase is given by $\theta_{f}$ and the matrix area fraction is given by $\theta_{m}$ with $\theta_{f}+\theta_{m}=1$. The graded material properties of the shaft are obtained by changing the local area fraction of fibers across the cross section of the shaft see Figure 1.

In what follows a constraint is placed on the total cross-sectional area occupied by the fibers. The goal of the design problem is to identify a graded distribution of fibers across 
the cross section such that the following requirements are met:

I. The reinforced shaft has a torsional rigidity that is acceptable.

II. The magnitude of the local point wise stress inside the composite is controlled over a designated subset of the cross section.

In Section 2 we describe a suitably formulated homogenized design problem that allows for the easy identification of fiber reinforced designs satisfying requirements (I) and (II). The homogenized formulation makes use of a multiscale stress criterion given in terms of the macrostress modulation function. The physical motivation for the multiscale stress criterion is presented in Section 2. The local effective properties and macrostress modulation function used to formulate the stress criterion are computed explicitly for the Hashin Shtrikman coated cylinder assemblage in [26]. These are given in terms of the elastic moduli of the matrix and fibers and the local area fraction of fibers. We point out that the coated cylinder assemblage gives the minimum amount of stress amplification among all periodic fiber microstructures with isotropic effective shear compliance [28]. A gradient based numerical implementation of the inverse homogenization design method is described in Section 3. In Section 4 computational results are given for the "X" shaped shaft cross section. This geometry typifies the junctions between composite substructures and possesses reentrant corners seen in lap joints and junctions of struts. We conclude by noting that the theoretical basis for the approach given here has been established for three dimensional structural design using multiphase locally periodic composites in the presence of point wise stress constraints see ([21], Theorems 5.1 and 5.2). For locally layered microstructures the corresponding theory is presented in [29].

\section{Homogenized design formulation and identification of op- timal graded fiber microgeometries.}

The inverse homogenization design method is a top down design approach. First a well posed homogenized design problem is developed. This design problem is given in terms of design variables that reflect the local microgeometry inside the composite. For the problem treated here the design variable for the homogenized design problem is given by the density function $\theta_{f}(\mathbf{x})$. The homogenized design problem is then solved to obtain an optimal density function. With the optimal density in hand we use it to recover an explicit graded fiber design that has structural properties close to that of the optimal homogenized design and satisfies prescribed point wise stress constraints. The homogenized design problem is described in the first subsection. The explicit link between homogenized designs and graded fiber reinforced designs that satisfy point wise stress constraints is provided in the second subsection.

\subsection{Homogenized design problem}

The design variable for the homogenized design problem is given by the density function $\theta_{f}(\mathbf{x})$. This function is interpreted as providing the local area fraction of the fiber phase in a homogenized design. The resource constraint on the fiber phase is given by

$$
\int_{\Omega} \theta_{f}(\mathbf{x}) d x_{1} d x_{2} \leq \Theta \times(\text { Area of } \Omega),
$$


where $0<\Theta<1$. At each point the local area fraction satisfies the box constraint given by

$$
0 \leq \theta_{f} \leq 1
$$

In this treatment the local fiber area fraction $\theta_{f}$ changes continuously with position according to the condition

$$
\left|\theta_{f}(\mathbf{x})-\theta_{f}(\mathbf{x}+\mathbf{h})\right| \leq K|\mathbf{h}|
$$

Here the constant $\mathrm{K}$ is prescribed by the designer. The universe of admissible designs given by all local area fractions $\theta_{f}$ satisfying the resource constraint, box constraints, and (2.3) is denoted by $D_{\Theta}$.

The compliance in shear for the matrix and fiber are given by $S_{m}=\left(2 G_{m}\right)^{-1}$ and $S_{f}=\left(2 G_{f}\right)^{-1}$ respectively. Here the matrix is more compliant and $S_{m}>S_{f}$. The effective shear compliance $S^{E}\left(\theta_{f}\right)$ for the Hashin Shtrikman coated sphere assemblage made from stiff fibers with area fraction $\theta_{f}$ is given by [16]

$$
S^{E}\left(\theta_{f}(\mathbf{x})\right)=S_{m}\left(\frac{S_{m}+S_{f}+\theta_{f}(\mathbf{x})\left(S_{f}-S_{m}\right)}{S_{m}+S_{f}+\theta_{f}(\mathbf{x})\left(S_{f}+S_{m}\right)}\right) .
$$

The macroscopic stress potential $\phi^{H}$ vanishes on the boundary of the shaft cross section and satisfies

$$
-\operatorname{div}\left(S^{E}\left(\theta_{f}\right) \nabla \phi^{H}\right)=1
$$

inside the cross section. The torsional rigidity for the homogenized shaft cross section made from a homogenized material with compliance $S^{E}\left(\theta_{f}\right)$ is given by

$$
\mathcal{R}\left(\theta_{f}\right)=2 \int_{\Omega} \phi^{H} d x_{1} d x_{2} .
$$

The macroscopic stress in the homogenized shaft is given by $\sigma^{H}=R \nabla \phi^{H}$ where $R$ is the rotation matrix associated with a counter clock wise rotation of $\pi / 2$ radians.

The multiscale stress criterion is given in terms of the macrostress modulation function introduced in [25]. The macrostress modulation function captures the interaction between the macroscopic stress $\sigma^{H}(\mathbf{x})$ and the microstructure. Consider the unit square $Q$ filled with Hashin Shtrikman coated cylinder assemblage with area fraction of fibers $\theta_{f}$. The coordinates of points inside the unit square are denoted by $\mathbf{y}$. The associated local shear compliance inside the unit square is denoted by $S\left(\theta_{f}, \mathbf{y}\right)$. Here $S\left(\theta_{f}, \mathbf{y}\right)=S_{f}$ in the fiber phase and $S\left(\theta_{f}, \mathbf{y}\right)=S_{m}$ in the matrix phase. In what follows all derivatives with respect to the microscopic variable $\mathbf{y}$ are denoted with subscripts. The microscopic response to the imposed macroscopic stress is given by $\sigma(\mathbf{x}, \mathbf{y})=R\left[\nabla_{\mathbf{y}}(w(\mathbf{x}, \mathbf{y}))+\nabla \phi^{H}(\mathbf{x})\right]$, where for each $\mathbf{x}$ in the shaft cross section $\Omega$ the $Q$ periodic fluctuating stress potential $w(\mathbf{x}, \mathbf{y})$ solves the microscopic equilibrium equation

$$
-\operatorname{div}_{\mathbf{y}}\left(S\left(\theta_{f}(\mathbf{x}), \mathbf{y}\right)\left(\nabla_{\mathbf{y}}(w(\mathbf{x}, \mathbf{y}))+\nabla \phi^{H}(\mathbf{x})\right)\right)=0, \mathbf{y} \text { in } Q .
$$

Here the $\mathbf{x}$ coordinate appears as a parameter. The relevant interaction is described by the macrostress modulation function $f\left(\theta_{f}, \sigma^{H}\right)$ given by

$$
f\left(\theta_{f}(\mathbf{x}), \sigma^{H}(\mathbf{x})\right)=\sup _{\mathbf{y} \operatorname{in} Q}\left\{|\sigma(\mathbf{x}, \mathbf{y})|^{2}\right\}
$$


Physically the macrostress modulation provides an upper envelope on the oscillating point wise local stress in the composite [25], [26].

The macrostress modulation is calculated explicitly for the Hashin Shtrikman coated cylinder assemblage in [26]. We define the amplification factor

$$
A\left(\theta_{f}\right)=\left(\frac{2 S_{m}}{S_{m}+S_{f}+\theta_{f}\left(S_{m}-S_{f}\right)}\right)^{2}
$$

and set

$$
f\left(\theta_{f}, \mathbf{v}\right)=A\left(\theta_{f}\right)|\mathbf{v}|^{2} \text { if } \theta_{f}>0 \text { and } f\left(\theta_{f}, \mathbf{v}\right)=|\mathbf{v}|^{2}, \text { if } \theta_{f}=0
$$

for every vector v. Here $A\left(\theta_{f}\right) \geq 1$ and $A(0)=\left(2 S_{m} /\left(S_{m}+S_{f}\right)\right)^{2}>1$. In the context of torsional rigidity the macrostress modulation is written in terms of the homogenized stress potential and is given by

$$
f\left(\theta_{f}(\mathbf{x}), \nabla \phi^{H}(\mathbf{x})\right) .
$$

It is pointed out that the Hashin Shtrikman coated cylinder assemblage gives the smallest amplification factor $A\left(\theta_{f}\right)$ among all locally periodic fiber microstructures with isotropic effective shear compliance [28].

We choose a subset $\mathcal{S}$ of the shaft cross section that lies a finite distance away from the boundary. On this set the prescribed multiscale stress criterion is given by

$$
f\left(\theta_{f}(\mathbf{x}), \nabla \phi^{H}(\mathbf{x})\right) \leq T^{2} .
$$

In this treatment domains with reentrant corners are considered so there will be a stress singularity at the corner. Therefore the choice of $T>0$ depends on the distance between $\mathcal{S}$ and the reentrant corner. It is clear that the multiscale stress criterion may not be satisfied by any homogenized design if $T$ is chosen too small.

The homogenized design problem is given by

$$
H P=\left\{\inf \left\{\mathcal{R}\left(\theta_{f}\right)\right\} ; \text { subject to: }\left\{\begin{array}{l}
\theta_{f} \text { in } D_{\Theta}, \\
f\left(\theta_{f}(\mathbf{x}), \nabla \phi^{H}(\mathbf{x})\right) \leq T^{2}, \text { for } \mathbf{x} \text { in } \mathcal{S}
\end{array}\right\}\right\} .
$$

The homogenized design problem $H P$ is well posed and there is an optimal design $\hat{\theta}_{f}$ provided $T$ is chosen large enough so that $D_{\Theta}$ contains at least one design, see [21].

\subsection{Identification of graded fiber design from the homogenized design}

In this subsection it is shown how to use the optimal design $\hat{\theta}_{f}$ of $H P$ to identify a graded fiber design satisfying the requirements (I) and (II). The building block for the microstructure is the unit cell filled with a Hashin Shtrikman coated cylinder assemblage [16]. The coated cylinder assemblage is constructed as follows. A space filling configuration of disks of different sizes ranging down to the infinitesimal is placed inside the unit period cell given by the unit square $Q$. Each disk is then partitioned into an annulus called the coating and a concentric disk which is a fiber cross section. The union of these "coated disks" make up the coated cylinder assemblage. The area fraction of the fiber phase is the same for all coated disks in the assemblage and is given by $\theta_{f}$. The union of the coatings comprises the 
matrix phase. The area fraction of the fiber phase for the coated cylinder assemblage inside the unit square $Q$ is easily seen to be $\theta_{f}$. The unit square filled with the coated cylinder assemblage is illustrated in Figure 1. A microstructure is obtained by rescaling the unit cell by the factor $\varepsilon$ so that it becomes the period cell for an $\varepsilon$ periodic composite.

In order to describe the graded fiber composite one partitions the shaft cross section $\Omega$ into the $N$ subdomains $\omega^{k}, k=1, \ldots, N$ and $\Omega=\cup_{k}^{N} \omega^{k}$. The maximum diameter of the subdomains in the partition is denoted by $\tau^{N}$. The partition is denoted by $\mathcal{P}^{\tau_{N}}$. A graded fiber composite is constructed by placing an $\varepsilon$ periodic Hashin Shtrikman coated cylinder geometry inside each subdomain $\omega^{k}$. The area fraction of fibers in each subdomain is given by the constant $\theta_{f}^{k}$ and these constants can change between subdomains. For future reference this type of locally periodic microstructure will be called a $\left(\tau_{N}, \varepsilon\right)$-graded Hashin Shtrikman fiber microstructure.

The local piecewise constant shear compliance for the $\left(\tau_{N}, \varepsilon\right)$-graded Hashin Shtrikman fiber microstructure is denoted by $S^{\varepsilon, N}$. The stress potential for this microstructure is denoted by $\phi^{\varepsilon, N}$ and vanishes on the boundary of the cross section. The stress potential satisfies the equilibrium equation

$$
-\operatorname{div}\left(S^{\varepsilon, N} \nabla \phi^{\varepsilon, N}\right)=1 .
$$

The torsional rigidity of the cross section is given by

$$
\mathcal{R}^{\varepsilon, N}=2 \int_{\Omega} \phi^{\varepsilon, N} d x_{1} d x_{2} .
$$

The nonzero components of the in plane stress are denoted by the vector $\sigma^{\varepsilon, N}=$ $\left(\sigma_{13}^{\varepsilon, N}, \sigma_{23}^{\varepsilon, N}\right)$ and are related to the gradient of the stress potential according to

$$
\sigma^{\varepsilon, N}=R \nabla \phi^{\epsilon, N}
$$

Here $R$ is the matrix corresponding to a counter clockwise rotation of $\pi / 2$ and $\left|\sigma^{\varepsilon, N}\right|=$ $\left|\nabla \phi^{\varepsilon, N}\right|$.

Given any partition $\mathcal{P}^{\tau_{N}}$ the partition $\mathcal{P}^{\tau_{\hat{N}}}$ is said to be a refinement of it, if $\tau_{\hat{N}}<\tau_{N}$ and if each subdomain belonging to $\mathcal{P}^{\tau_{\hat{N}}}$ is a subset of a subdomain in $\mathcal{P}^{\tau_{N}}$. Next for any given partition $\mathcal{P}^{\tau_{N}}$ we introduce a nested sequence of refinements $\left\{\mathcal{P}^{\tau_{N} \ell}\right\}_{\ell=1}^{\infty}$ such that $\mathcal{P}^{\tau_{N}^{1}}=\mathcal{P}^{\tau_{N}}$ and $\mathcal{P}^{\tau_{N}^{\ell+1}}$ is a refinement of $\mathcal{P}^{\tau_{N} \ell}$ with $\lim _{\ell \rightarrow \infty} \tau_{N^{\ell}}=0$. Here we assume $\varepsilon<\tau_{N^{\ell}}$ and we will consider the sequence of $\left(\tau_{N^{\ell}}, \varepsilon\right)$-graded Hashin Shtrikman fiber microstructures with associated stress potentials $\phi^{\varepsilon, N^{\ell}}(\mathbf{x})$.

The identification of a graded fiber composite satisfying the point wise stress constraints is given in the following Proposition.

\section{Proposition 2.1. Identification of graded microstructure.}

Consider a homogenized design specified by $\theta_{f}$ in $D_{\Theta}$ such that the multiscale stress criterion (2.12) holds on the subset $\mathcal{S}$ of the shaft cross section. Consider also any partition $\mathcal{P}^{\tau_{N}}$ of the design domain $\Omega$. Then for any given $t>T$ and small number $\delta>0$, there is a refinement $\mathcal{P}^{\tau_{N} \ell}$ of the partition and an associated $\left(\tau_{N^{\ell}}, \varepsilon\right)$-graded Hashin Shtrikman fiber microstructure for which the part of $\mathcal{S}$ over which the stress constraint

$$
\left|\nabla \phi^{\varepsilon, N^{\ell}}(\mathbf{x})\right| \leq t
$$


is violated has measure (area) less than $\delta$ and

$$
\left|\mathcal{R}^{\varepsilon, N^{\ell}}-\mathcal{R}\left(\theta_{f}\right)\right|<\delta,
$$

and

$$
\sum_{k=1}^{N^{\ell}}\left|\omega^{k}\right| \theta_{f}^{k} \leq \Theta \times(\text { Area of } \Omega)+\delta .
$$

Here $\left|\omega^{k}\right|$ denotes the area of $\omega^{k}$. Moreover the area fractions $\theta_{f}^{k}$ are determined from $\theta_{f}$ through the averages given by

$$
\theta_{f}^{k}=\frac{1}{\left|\omega^{k}\right|} \times \int_{\omega^{k}} \theta_{f}(\mathbf{x}) d x_{1} d x_{2} .
$$

This Proposition is established in [21]. The homogenized design formulation together with Proposition 2.1 provide an inverse homogenization method for identifying microstructures that satisfy point wise stress constraints while delivering a torsional rigidity close to that given by the optimal design $\hat{\theta}_{f}$ for the homogenized design problem.

\section{Gradient algorithm for the homogenized design problem}

In the computational examples we enforce the stress constraint by adding a penalty term to the torsional rigidity and minimize

$$
L\left(\theta_{f}\right)=-\mathcal{R}\left(\theta_{f}\right)+l \int_{\Omega}\left(f\left(\theta_{f}, \nabla \phi^{H}\right)\right)^{p} d x_{1} d x_{2},
$$

over all $\theta_{f}$ in $D_{\Theta}$ where $l>0$ and $\phi^{H}$ satisfies

$$
-\operatorname{div}\left(S^{E}\left(\theta_{f}\right) \nabla \phi^{H}\right)=1
$$

and vanishes at the boundary. The computational examples provided here will be carried out for a domain with reentrant corners of interior angle $3 \pi / 2$. In view of the strength of the associated singularity at the reentrant corners the power " $p$ " appearing in the penalty term is chosen to be less than 3 . This minimization problem is well posed and one can find a minimizing density $\hat{\theta}_{f}(\mathbf{x})$ see $[21]$.

Given the minimizing density and associated stress potential $\hat{\phi}^{H}$ one considers sets of the form

$$
A_{T}=\left\{\mathbf{x} \in \Omega: f\left(\hat{\theta}_{f}(\mathbf{x}), \nabla \hat{\phi}^{H}(\mathbf{x})\right) \leq T^{2}\right\}
$$

For fixed choices of $\delta>0$ and $t>T$ Proposition 2.1 can be used to recover a $\left(\tau_{\hat{N}}, \varepsilon\right)$-graded Hashin Shtrikman fiber microstructure for which the the part of $A_{T}$ over which the stress constraint

$$
\left|\nabla \phi^{\varepsilon, \hat{N}}(\mathbf{x})\right| \leq t
$$

is violated has measure less than $\delta$,

$$
\left|\mathcal{R}^{\varepsilon, \hat{N}}-\mathcal{R}\left(\hat{\theta}_{f}\right)\right|<\delta
$$


and

$$
\sum_{k=1}^{\hat{N}}\left|\omega^{k}\right| \hat{\theta}_{f}^{k} \leq \Theta \times(\text { Area of } \Omega)+\delta .
$$

Here the area fractions $\hat{\theta}_{f}^{k}$ are given by

$$
\hat{\theta}_{f}^{k}=\frac{1}{\left|\omega^{k}\right|} \times \int_{\omega^{k}} \hat{\theta}_{f}(\mathbf{x}) d x_{1} d x_{2} .
$$

To proceed numerically we compute the derivative of the objective function given by (3.1) with respect to the design variable $\theta_{f}$. The derivative will be used to develop a gradient minimization algorithm. However we note that the macrostress modulation function (2.10) is discontinuous at $\theta_{f}=0$. In order to proceed we restrict $\theta_{f}$ to satisfy the constraint $0<\theta_{f}^{\min } \leq \theta_{f} \leq 1$ at each point in the design domain. For our computations we choose $\theta_{f}^{\min }=0.01$. For this choice the modulation function is differentiable with respect to the density and we proceed to develop a gradient method subject to these new constraints.

The gradient of the objective is computed with the help of an adjoint field $\lambda$. Here $\lambda$ is the solution of

$$
-\operatorname{div}\left(S^{E}\left(\theta_{f}\right) \nabla \lambda\right)=1+l \operatorname{div}\left(2 p\left(A\left(\theta_{f}\right) \nabla \phi^{H} \cdot \nabla \phi^{H}\right)^{p-1} A\left(\theta_{f}\right) \nabla \phi^{H}\right) .
$$

where $1 \leq p<3$ and $\lambda=0$ on the boundary. For $\eta<<1$ the change in the stress potential $\phi^{H}$ due to small local perturbations $\eta \tilde{\theta}_{f}$ in the area fraction, is written as $\tilde{\phi}$ and

$$
-\operatorname{div}\left(S^{E}\left(\theta_{f}\right) \nabla \tilde{\phi}\right)=\operatorname{div}\left(\left(\partial_{\theta_{f}} S^{E}\left(\theta_{f}\right) \tilde{\theta}_{f}\right) \nabla \phi^{H}\right)
$$

where $\tilde{\phi}=0$ on the boundary. Applying standard arguments using (3.2), (3.8) and (3.9) one calculates to first order that

$$
\begin{aligned}
\triangle L & =\eta \int_{\Omega} \partial_{\theta_{f}} L \tilde{\theta}_{f} d x_{1} d x_{2}, \quad \text { where } \\
\partial_{\theta_{f}} L & =\partial_{\theta_{f}} S^{E}\left(\theta_{f}\right) \nabla \lambda \cdot \nabla \phi^{H}+2 \operatorname{lp}\left(A\left(\theta_{f}\right) \nabla \phi^{H} \cdot \nabla \phi^{H}\right)^{p-1} \partial_{\theta_{f}} A\left(\theta_{f}\right) \nabla \phi^{H} \cdot \nabla \phi^{H}(3.1
\end{aligned}
$$

The continuity constraints on $\theta_{f}(\mathbf{x})$ expressed by (2.3) are enforced by the way in which the design variable is initialized and updated. The local average of a scalar function $f$ over the disk of radius $R$ centered at $\mathbf{p}$ is denoted by $<f>^{R}(\mathbf{p})$. For a given field $\theta_{f}$ satisfying the resource constraint (2.1) and box constraint $\theta_{f}^{\text {min }} \leq \theta_{f} \leq 1$ the initial choice of design variable $\theta_{f}^{0}$ is given by

$$
\theta_{f}^{0}=\left\langle\theta_{f}\right\rangle^{R}(\mathbf{x})
$$

At the $\mathrm{n}$ th step we suppose that $\theta_{f}^{n}$ is given and we solve for $\phi^{H}$ and $\lambda$ using the system of equations (3.2) and (3.8). The design variable $\theta_{f}^{n}$ is updated according to

$$
\theta_{f}^{n+1}=\left\langle\theta_{f}^{n}-\eta \partial_{\theta_{f}} L\right\rangle^{R}(\mathbf{x})
$$

were $\partial_{\theta_{f}} L$ is given by (3.10). If the right hand side of (3.12) lies out side the box constraint $\theta_{f}^{\min } \leq \theta_{f} \leq 1$ we update according to

$$
\begin{aligned}
\theta_{f}^{n+1} & =\theta_{f}^{\min }, \text { if }\left\langle\theta_{f}^{n}-\eta \partial_{\theta_{f}} L\right\rangle^{R}(\mathbf{x})<\theta_{f}^{\min } \text { and } \\
\theta_{f}^{n+1} & =1, \text { if }\left\langle\theta_{f}^{n}-\eta \partial_{\theta_{f}} L\right\rangle^{R}(\mathbf{x})>1 .
\end{aligned}
$$


Because the updated functions are given by averages of bounded functions it is easily seen that they satisfy (2.3) for a non-negative constant $K$ independent of $\mathbf{x}$. The use of local averaging in the update scheme is similar to the use of filters in topology optimization see $[5]$ and $[6]$.

For points near the boundary a difficulty arises when defining the averages. This is dealt with by extending $\theta_{f}$ to the slightly larger domain $\Omega_{R}=\left\{\mathbf{x}\right.$ in $\left.\mathbf{R}^{2} ; \operatorname{dist}(\mathbf{x}, \Omega) \leq R\right\}$. The particular form of extension is up to the designer. Possibilities include setting $\theta_{f}=1$ in $\Omega_{R} \backslash \Omega$ or reflection of $\theta_{f}$ across the boundary of $\Omega$ into $\Omega_{R}$. In the discretized problem used for the simulations we allow $\theta_{f}$ to take constant values inside each element and define $\left\langle\theta_{f}\right\rangle^{R}$ to be the average of $\theta_{f}$ taken over all neighboring elements.

\section{Numerical results for the $\mathrm{X}$-shaped cross section}

The computational examples are carried out for an "X" shaped domain. All interior angles for the reentrant corners are fixed at $3 \pi / 2$ radians. The shear stiffness of the matrix is assigned the value $G_{m}=1 G P a$ and the shear stiffness of the fiber phase is assigned the value $G_{f}=2 G P a$. For these choices $S_{m}=1 /\left(2 G_{m}\right)=0.5$ and $S_{f}=1 /\left(2 G_{f}\right)=0.25$. All of the design optimizations are carried out with the matrix material occupying $70 \%$ of the shaft cross section. The local fiber area fraction $\theta_{f}$ is constrained to lie in the interval $0.01 \leq \theta_{f} \leq 1$.

In the first example we optimize for torsional rigidity only. The resulting optimal design is referred to as design 1 . The grey scale plot of the local area fraction of matrix material $\hat{\theta}_{m}=1-\hat{\theta_{f}}$ is given in Figure 2. Here the lightest regions correspond to points where $\hat{\theta}_{m}=0$. The darkest regions correspond to points where $\hat{\theta}_{m}=0.99$. As expected this design ignores the stress concentration at the reentrant corners and a zone of stiff material surrounds a more compliant inner core and lies adjacent to the reentrant corners. In the next example the torsional rigidity is optimized in the presence of an integral penalization $\int(f)^{1}$, i.e., $p=1$ for the Lagrangian in (3.1). The resulting design is referred to as design 2. The plot of $\hat{\theta}_{m}$ for this design is given in Figure 3. For this case it is seen that there is a topology change in the design and the more compliant material now surrounds the stiffer material shielding it from the stress concentration at the reentrant corners. In the final example the torsional rigidity is optimized in the presence of a stronger integral penalization given by $\int(f)^{2}$, i.e., $p=2$ for the Lagrangian in (3.1). The resulting design is referred to as design 3. The plot of $\hat{\theta}_{m}$ for this design is given in Figure 4 . As in design 2 it is seen that the more compliant material shields the stiff material from the stress concentration at the reentrant corners. The associated torsional rigidities for each of these cases are listed in Table 1. It is seen from the table that the torsional rigidity drops by $44 \%$ for the stress constrained designs.

The contour plot of the macrostress modulation function $f$ for design 1 is given in Figure 5. Figures 6 and 7 give the contour plots for $f$ in designs 2 and 3 respectively. When comparing designs 1, 2 and 3 it is clear from Figures 5, 6 and 7 that designs 2 and 3 provide a significant reduction in the size of the over stressed zone $f \geq 1$.

It is pointed out that Proposition 2.1 provides the method for constructing a $\left(\tau_{N}, \varepsilon\right)$ graded Hashin Shtrikman fiber microstructure from the data given in design 2. To fix ideas we choose a tolerance $\delta=1 / 1000$ and $t=1.001$. Then Proposition 2.1 shows how to construct a $\left(\tau_{N}, \varepsilon\right)$-graded Hashin Shtrikman fiber microstructure with torsional rigidity 
$\mathcal{R}^{\varepsilon, N}$ satisfying

$$
\left|\mathcal{R}^{\varepsilon, N}-0.204\right|<1 / 1000
$$

and for which the magnitude of the in plane stress lies below 1.001 for all points in the region $f<1$ of Figure 6 , with the possible exception of a subset of points of area less than $1 / 1000$.

\section{Acknowledgments.}

This research effort is sponsored by NSF through grant DMS-0406374 and by the Air Force Office of Scientific Research, Air Force Materiel Command USAF, under grant numbers F49620-02-1-0041 and FA9550-05-1-0008. The US Government is authorized to reproduce and distribute reprints for governmental purposes notwithstanding any copyright notation thereon. The views and conclusions herein are those of the authors and should not be interpreted as necessarily representing the official policies or endorsements, either expressed or implied of the Air Force Office of Scientific Research or the US Government.

\section{References}

[1] G. Allaire, R.V. Kohn, Optimal design for minimum weight and compliance in plane stress using extremal microstructure, Euro. J. Mech. 12 (1993) 839-878.

[2] G. Allaire, Shape Optimization by the Homogenization Method, Springer, New York 2002 .

[3] G. Allaire, F. Jouve, H. Mallot, Topology optimization for minimum stress design with the homogenization method, Struct. Multidisc. Optim. 28 (2004) 87-98.

[4] M.P. Bendsoe, N. Kikuchi, Generating optimal topologies in structural design using a homogenization method, Comput. Methods Appl. Mech. Engrg. 71 (1988) 197-224.

[5] M.P. Bendsoe, O. Sigmund, Topology Optimization, Theory, Methods and Applications, Springer, Berlin 2003.

[6] B. Bourdin, Filters in topology optimization, Int. J. Numer. Meth. Engng. 50 (2001) $2143-2158$.

[7] K.T. Cheng, N. Olhoff, An investigation concerning optimal design of solid elastic plates, Internat. J. Solids and Structures 17 (1981) 305-323.

[8] A. Cherkaev, R.V. Kohn, Topics in the Mathematical Modelling of Composite Materials, Progress in Nonlinear Differential Equations and their Applications, Birkhauser, Boston, 1997.

[9] A. Cherkaev, Variational Methods for Structural Optimization, Springer, New York, 2000. 
[10] A. Cherkaev, R. Palais, Optimal design of three-dimensional axisymmetric elastic structures. Structural Dynamic Systems Computational Techniques and Optimization, Gordon and Breach Int. Ser. Eng. Technol. Appl. Sci., vol.9, Gordon and Breach, Amsterdam, 1999, pp. 237-267.

[11] A.R. Diaz, N. Kikuchi, Solutions to shape and topology eigenvalue optimization problems using a homogenization method, Int. Num. Meth. Eng. 35 (1992) 1487-1502.

[12] A.R. Diaz, R. Lipton, Optimal material layout for three-dimensional elastic structures subject to multiple loads, Mech. Struct. and Mach. 28 (2000) 219-236.

[13] P. Duysinx, M.P. Bendsoe, Topology optimization of continuum structures with local stress constraints, Int. J. Num. Meths. Eng. 43 (1998) 1453-1478.

[14] D. Fujii, B.C. Chen, N. Kikuchi, Composite material design of two-dimensional structures using the homogenization design method, Internat. J. Numer. Methods Engrg. 50 (2001) 2031-2051.

[15] Y. Grabovsky, Optimal design problems for two-phase conducting composites with weakly discontinuous objective functionals, Advances in Applied Mathematics, 27 (2001) 683-704.

[16] Z. Hashin, S. Shtrikman, A variational approach to the theory of the effective magnetic permeability of multiphase materials, J. Appl. Phys. 33 (1962) 3125-3131.

[17] C.S. Jog, R.B. Haber, M.P. Bendsoe, Topology design with optimized, self-adaptive materials, Internat. J. Numer. Methods Engrg. 37 (1994) 1323-1350.

[18] R.V. Kohn, G. Strang, Optimal Design and Relaxation of Variational Problems, Communications on Pure and Applied Mathematics 34 (1986) Part I, 113-137, Part II, 139-182, Part III, 357-377.

[19] T. Lewinski, J.J. Telega, Plates, Laminates and Shells. Asymptotic Analysis and Homogenization, World Scientific, Singapore 2000.

[20] R. Lipton, Design of functionally graded composite structures in the presence of stress constraints, International Journal of Solids and Structures 39 (2002) 2575-2586.

[21] R. Lipton, Homogenization and design of functionally graded composites for stiffness and strength, in Nonlinear Homogenization and its Application to Composites, Polycrystals, and Smart Materials, Edited by P. Ponte Castaneda and J.J. Telega, NATO Science series II Mathematics, Physics, and Chemestry, vol. 170, Springer Verlag, Berlin, 2004, pp. 169-192.

[22] R, Lipton and A. Velo, Optimal design of gradient fields with applications to electrostatics, in Nonlinear Partial Differential Equations and Their Applications: College de France Seminar vol. XIV, Studies in Mathematics and its Applications, North Holland, Elsevier, 2002, pp. 509-532

[23] R. Lipton, Relaxation through homogenization for optimal design problems with gradient constraints, J. Optim. Theory Appl. 114 (2002) 27-53. 
[24] R. Lipton, Stress constrained G closure and relaxation of structural design problems, Quarterly of Applied Mathematics 62 (2004) 295-321.

[25] R. Lipton, Assessment of the local stress state through macroscopic variables, Phil. Trans. R. Soc. Lond. A. 361 (2003) 921-946.

[26] R. Lipton, Bounds on the distribution of extreme values for the stress in composite materials, J. Mech. Phys. Solids 52 (2004) 1053-1069.

[27] R. Lipton, Homogenization theory and the assessment of extreme field values in composites with random microstructure, SIAM J. on Appl. Math. 65 (2004) 475-493.

[28] R. Lipton, Optimal lower bounds on the electric-field concentration in composite media, Journal of Applied Physics 96 (2004) 2821-2827.

[29] R. Lipton, M. Stuebner, Optimal design of graded microstructure through inverse homogenization for control of pointwise stress, submitted for publication.

[30] K. Lurie, Applied Optimal Control Theory of Distributed Systems, Plenum Press, New York 1993.

[31] K.A. Lurie, A.V. Cherkaev, Effective characteristics of composite materials and the optimal design of structural elements, Uspekhi Mekhaniki (Advances in Mechanics) 9 (1986) $3-81$.

[32] F. Murat, L. Tartar, Calcul des variations et homogénéisation: Les Méthodes de l'Homogénéisation: Théorie et Applications en Physique, Ecole d'Eté d'Analyse Numérique C.E.A.-E.D.F.-INRA (Bréau-sans-Nappe, 1983), Collection de la Direction des Études et Recherches d'Electricité de France vol. 57, Eyrolles, Paris, 1985, pp. 319-369.

[33] N. Olhoff, On optimum design of structures and materials, Meccanica 31 (1996) 143161.

[34] P. Pedregal, Fully Explicit Quasiconvexification of the Square of the Gradient of the State in Optimal Design, Electronic Research Announcements of the American Mathematical Society 7 (2001) 72-78.

[35] O. Sigmund, S. Torquato, Design of materials with extreme thermal expansion using a three-phase topology optimization method, J. Mech. Phys. Solids 45 (1997) 1037-1067.

[36] L. Tartar, Remarks on optimal design problems, in Calculus of Variations, Homogenization and Continuum Mechanics, Edited by G. Buttazzo, G. Bouchitte, and P. Suquet, World Scientific, Singapore, 1994, pp. 279-296.

[37] L. Tartar, An introduction to the homogenization method in optimal design, Springer Lecture Notes in Mathematics, vol. 1740, Springer, Berlin, 2000, pp. 45-156.

[38] A. Velo, Optimal Design of Gradient Fields with Applications to Electrostatics, Ph.D. Thesis, Department of Mathematical Sciences Worcester Polytechnic Institute, 2000. 


\begin{tabular}{|c|c|c|c|}
\hline Design \# & Stress Constraint & Matrix-Volume Fraction & Torsional Rigidity \\
\hline 1 & None & $70 \%$ & 0.368 \\
\hline 2 & $\int(f)^{1}$ & $70 \%$ & 0.204 \\
\hline 3 & $\int(f)^{2}$ & $70 \%$ & 0.204 \\
\hline
\end{tabular}

Table 1: 


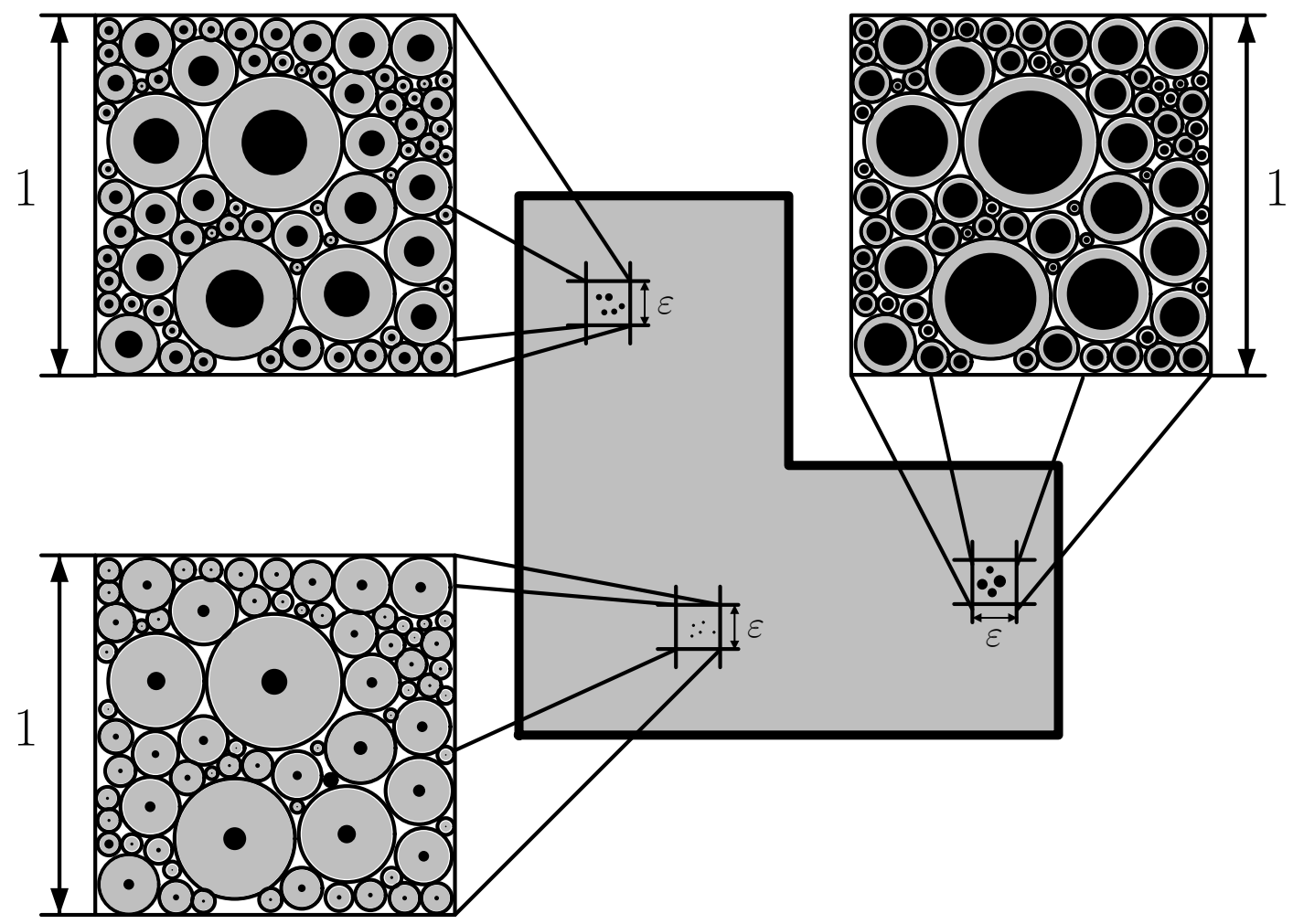

Figure 1: Cross section of shaft filled with a graded locally periodic Hashin Shtrikman coated cylinder assemblage. The change in the local fiber area fraction across the structure is illustrated. 


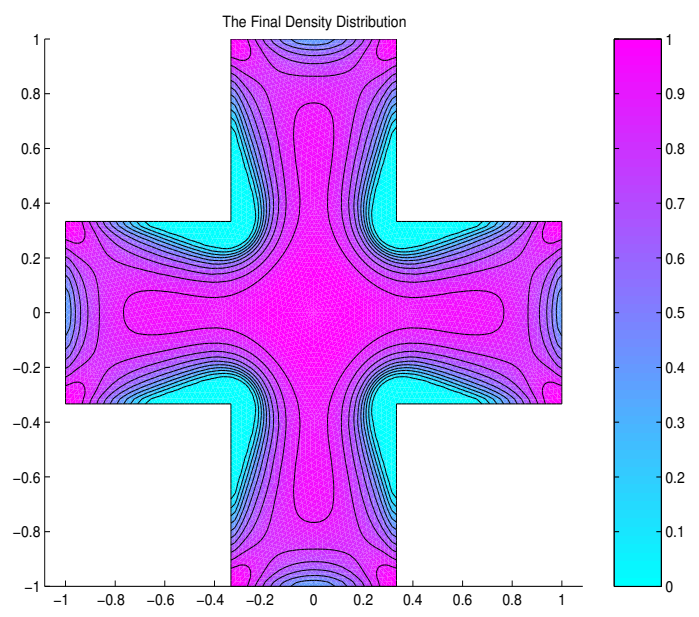

Figure 2: Design 1. Grey scale plot of the area fraction of matrix material inside the Xshaped shaft cross section optimized for torsional rigidity only. Lightest regions are made exclusively of stiff fiber-phase material. Darkest regions contain only $1 \%$ by area fraction of fiber phase material.

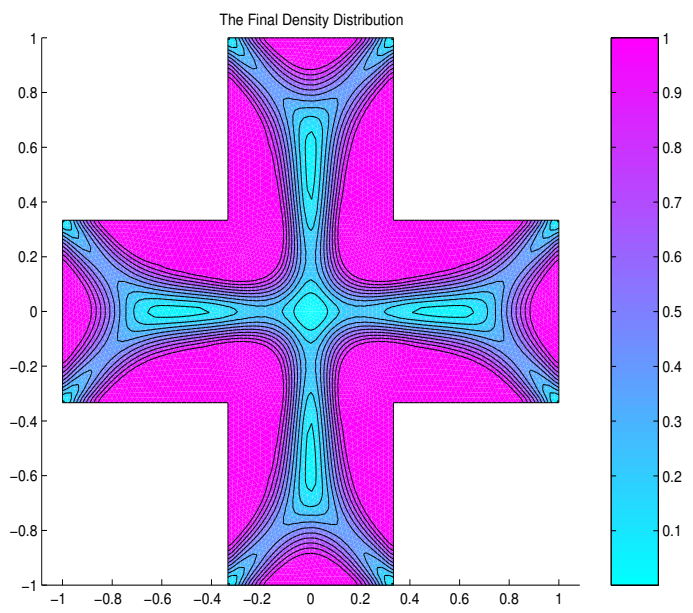

Figure 3: Design 2. Area fraction distribution of matrix material inside the $\mathrm{X}$-shaped cross section optimized for torsional rigidity with $p=1$ integral penalty on $f$. 


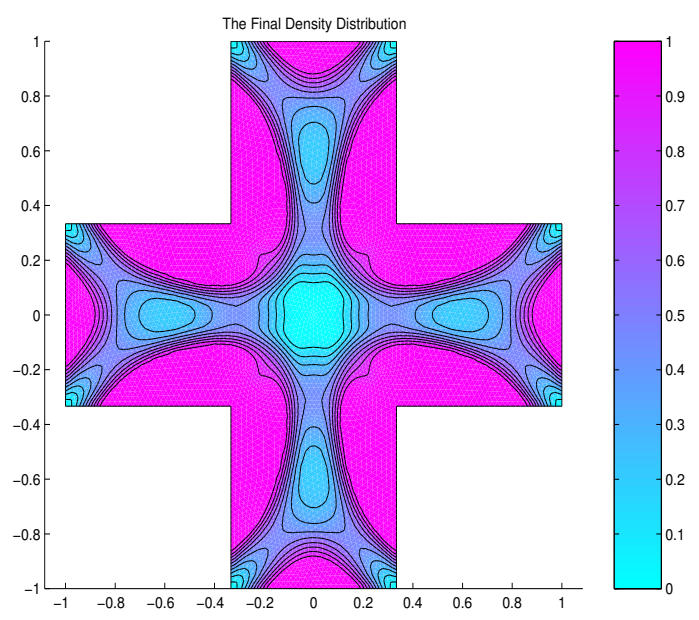

Figure 4: Design 3. Area fraction distribution of matrix material inside the X-shaped cross section optimized for torsional rigidity with $p=2$ integral penalty on $f$.

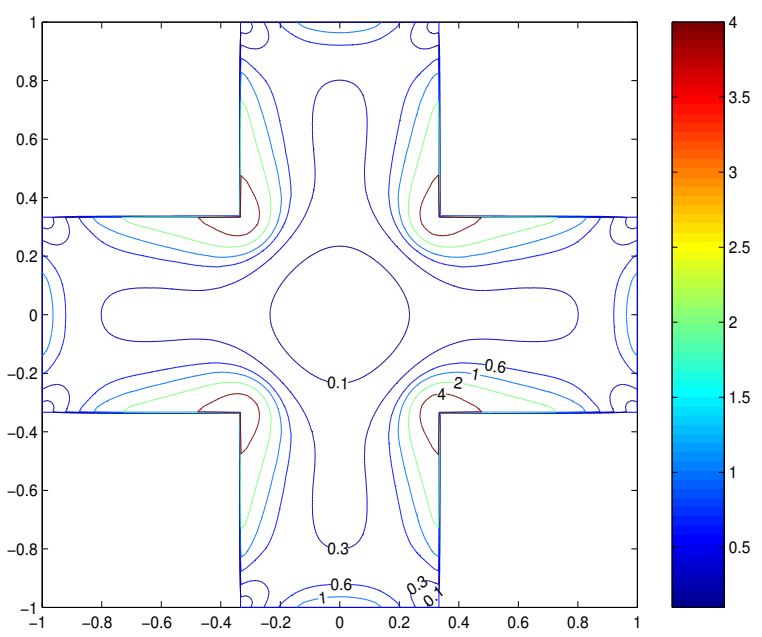

Figure 5: Contour plot of $f$ for design 1. 


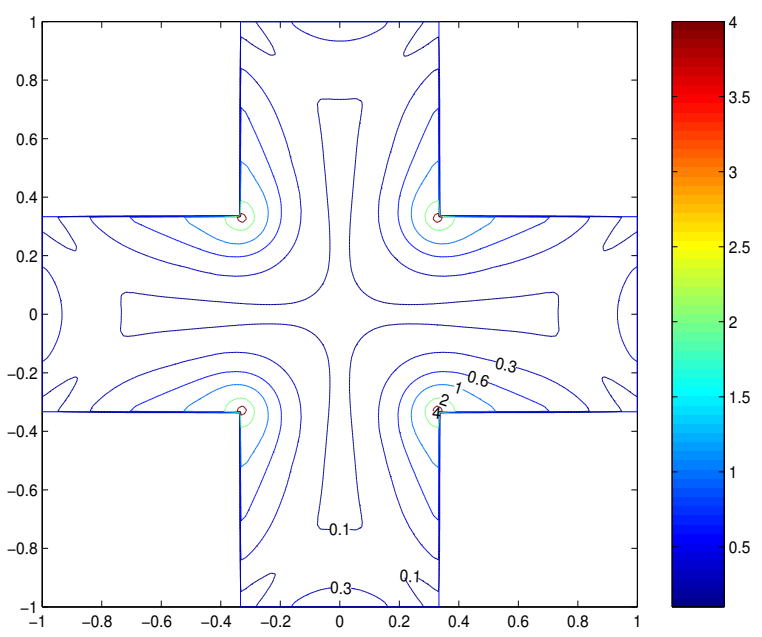

Figure 6: Contour plot of $f$ for design 2.

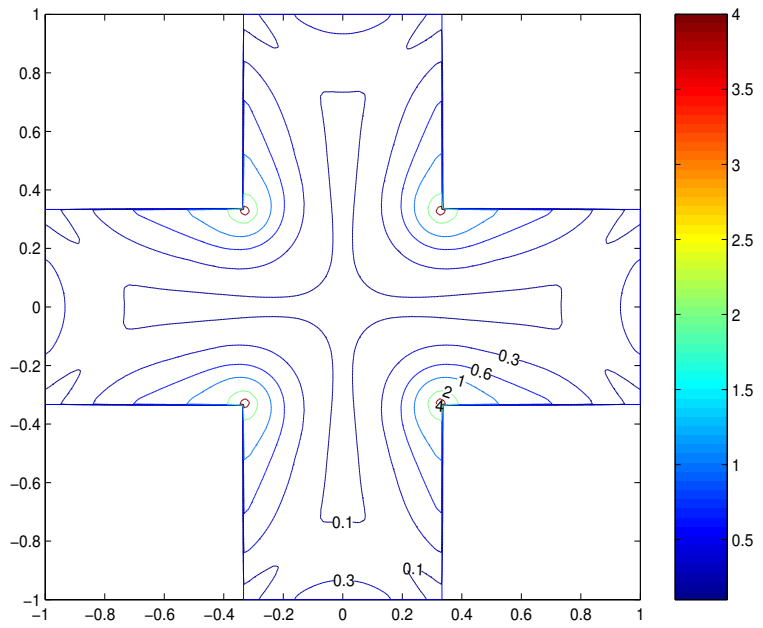

Figure 7: Contour plot of $f$ for design 3. 